\title{
A note regarding conditioning simulation trials for data-poor management strategy evaluations
}

\author{
ANDRÉ E. PUNT ${ }^{1,2}$ \\ Contacte-mail: aepunt@uw.edu
}

\begin{abstract}
Management Strategy Evaluation is a key tool used by the Scientific Committee of the International Whaling Commission to identify appropriate management strategies for commercial and aboriginal subsistence whaling. In several cases, Bayesian approaches have been used to condition the operating models and the assumed priors may substantially impact the conclusions regarding appropriate management strategies in data-poor situations. Three approaches for defining a prior on the 'scaling' parameter (on 'initial depletion', 'current depletion', and 'carrying capacity') are compared in terms of the proportion of draws from the prior that are rejected during the construction of the post-model-pre-data distribution and hence the resulting implied distribution for initial and current depletion. Overall, placing a prior on initial depletion leads to the fewest rejected draws but the implied distribution for current depletion depends on the catch history. In contrast, placing a prior on carrying capacity leads to less optimistic distributions for initial and current depletion and perhaps to unreasonably optimistic distributions for MSY. The issue of the appropriate distribution for the 'scaling' parameter in population models should be an explicit component of Management Strategy Evaluation and Bayesian assessments in data-poor situations in general.
\end{abstract}

KEYWORDS: BAYESIAN METHODS; MANAGEMENT STRATEGY EVALUATION; WHALING

\section{INTRODUCTION}

The Scientific Committee of the International Whaling Commission (IWC) uses Management Strategy Evaluation $(\mathrm{MSE})^{3}$ to compare the abilities of alternative candidate management strategies to achieve pre-specified management goals for commercial and aboriginal subsistence whaling recognising the inherent scientific uncertainty (Punt and Donovan, 2007; Punt et al., 2016). The IWC was the first organisation to make extensive use of MSE, but MSE is now considered state of the art by most marine renewable resource management bodies. The MSE approach has been used to evaluate management strategies for fish and invertebrate resources (e.g. Plagányi et al., 2007; Hillary et al., 2016) as well as in a broader ecosystem context (e.g. Dichmont et al., 2013; Fulton et al., 2919), and has been proposed for use in the terrestrial realm (Bunnefeld et al., 2011).

A key component of MSE is the operating model, which is a mathematical model of the system being managed, its current status and how future data will be generated. The values for the parameters of the operating model are specified (or the operating model is 'conditioned') by fitting it to the available data. In general, the operating models used to compare alternative variants of the Revised Management Procedure (RMP; IWC, 2012) are conditioned using a bootstrap-like procedure (e.g. western North Pacific Bryde's whales: IWC, 2019a; North Pacific minke whales: IWC, 2014a; North Atlantic minke whales: IWC, 2017). In contrast, the operating models used to compare alternative candidate Strike Limit Algorithms for aboriginal subsistence whaling have often been fitted using Bayesian methods (e.g. Bering-Chukchi-Beaufort Seas [B-C-B] bowhead whales:

${ }^{3}$ Usually termed the Management Procedure approach in IWC literature.
IWC, 2003; West Greenland bowhead, humpback and fin whales: IWC, 2014b, IWC, 2019b) ${ }^{4}$.

Bayesian methods allow (and in fact require) prior distributions to be imposed on the parameters of the population dynamics model, with these updated into posterior distributions based on the available data. The (estimated) parameters of the operating model relate to biological parameters (survival, age-at-maturity, the oldest age at which the juvenile survival rate applies), productivity (usually quantified by the Maximum Sustainable Yield rate; MSYR), current abundance $\left(N_{\text {cur }}\right)$, and a parameter that determines the current status of the population relative to carrying capacity (the 'scaling' parameter). The last three parameters have the greatest influence on the performance of candidate management strategies, which has led most IWC MSEs to develop trials in which MSYR is set to default values agreed by the Scientific Committee after considerable review (IWC, 2014c) rather than being treated as estimable (often MSYR $_{1+}=1 \%$ and MSYR ${ }_{\text {mat }}=4 \%$ ). The prior for current abundance is usually treated as 'informative' and set to the sampling distribution for a recent estimate of abundance (equivalent to placing a uniform prior on current (year $\mathrm{y}_{\text {cur }}$ ) abundance and including the recent estimate of abundance in the likelihood function). The 'scaling' parameter has usually been taken to be carrying capacity $(K)$, with the prior for $K$ taken to be uniform over a suitably broad range.

This paper explores options for choosing the prior for the scaling parameter. It would be desirable for this choice to be irrelevant, as would be the case for a stock such as the

${ }^{4}$ Exceptions are the most recent trials for the Eastern North Pacific gray whales (maximum likelihood approach; IWC, 2019c) and West Greenland minke whales (based on the operating model used to evaluate RMP variants). 
B-C-B bowheads for which the available data are fairly informative (but cannot place an upper limit on [present] carrying capacity). Desirable properties of this parameter are that (a) a wide range of scenarios are represented in the trials that would be conducted if there were no data, and (b) the posterior for the parameter closely matches the prior if there were no data (i.e. the specified prior is actually used as expected by those developing trials; A.E. Punt, pers. obs.) and hence relatively few of the draws from the priors are rejected because the population model is inconsistent with the generated values for the parameters. For example, an inconsistency would arise if the catch series was a constant 100 whales annually, the value for carrying capacity was set to 1,000 and the value for current population size to 999 unless the population growth rate parameter was set to a very (unrealistically) high value. Ideally, the set of parameters from the posterior should be such that the true population trajectory is included within the set of implied population trajectories.

The focus for this paper is the case in which the population projections do not start at carrying capacity but at some lower value in some year $\mathrm{y}_{\mathrm{I}}$, as is the case for all the recent operating models on which evaluations of Strike Limits Algorithms are based (IWC, 2014b; 2019). The paper considers the following three (almost exhaustive) options for defining the scaling parameter:

(A) The scaling parameter is taken to be the ratio of abundance in year $\mathrm{y}_{\mathrm{I}}$ (year 25 for this paper) relative to carrying capacity ('initial depletion') $D_{\mathrm{I}}$, with the prior for initial depletion assumed to be uniform over 0.01 to 0.99 , i.e. $D_{\mathrm{I}} \sim \mathrm{U}[0.01,0.99]$, with carrying capacity for each draw from the prior set such that current abundance equals a value drawn from the sampling distribution for $N_{\text {cur }}$ (denoted 'Year 25 depletion prior');

(B) The scaling parameter is taken to be current $\left(\mathrm{y}_{\mathrm{Cur}}\right.$; year 75 for this paper) depletion, with the prior for current depletion assumed to be uniform over 0.01 to 0.99 , i.e. the implied prior for current abundance $N_{\text {cur }}$ is uniform between 1 and $99 \%$ of carrying capacity. This assumption leads to the following prior for carrying capacity $K \sim U\left[\tilde{N}_{\text {cur }} / 0.99, \tilde{N}_{\text {cur }} / 0.01\right]$ where is $\tilde{N}_{\text {cur }}$ is the mean of sampling distribution for current abundance (denoted 'Carrying capacity prior (A)'); and

(C) The scaling parameter is taken to be carrying capacity, $K$, with a uniform prior over a 'wide range' placed on the logarithm of $K$, illustrated by $K \sim U\left[\ell \mathrm{n} \tilde{N}_{\text {cur }}\right.$ $\left.\ln \left(100 \tilde{N}_{\text {cur }}\right)\right]$ (denoted 'Carrying capacity prior (B)').

The last two options are based on the same support range for carrying capacity.

\section{METHODS}

\section{Overview of the testing procedure}

Two analyses (one that ignores observation error and one that accounts for observation error) are conducted to compare the three approaches for specifying the scale parameter (A-C above). All of these assume that catches started in year 0 and continued to year 75, and that the catches from years 25 to 75 are known. This reflects a case where the earlier catches are either not known well or there is concern that the abundance changed over years 0 to 25 for reasons not captured by the known catch history (e.g. if the stock is found in a region where catches are taken, but they are not included in the catch history for the stock; e.g. fin whales off West Greenland, or carrying capacity changed). The stock is assumed to be 1,000 animals at the start of year 75 (i.e. $\left.N_{75}=1,000\right)$, although the methods are such that the results are independent of the choice of the value for $N_{75}$.

The two analyses are:

(1) Conduct projections from year 25 to year 75 where the abundance in year 75 is known to be 1,000, for 99 values of the 'scaling' parameter (initial depletion or carrying capacity) across its assumed plausible range (this is referred to as a 'hitting' analysis within the IWC Scientific Committee).

(2) As for (1), except that 1,000 values for $N_{75}$ are drawn from a prior (lognormal with mean 1,000 and standard error of the $\log 0.3^{5}$ ) rather than $N_{75}$ being known to be 1,000 (the 'post-model-pre-data distribution' analysis ${ }^{6}$ ).

Results are shown for three values for $N_{75} / K(0.25,0.5$ and 0.75 ) for each of five effort series (Fig. 1, left column; resulting catches in Fig. 1, columns 2-4). The results of the first analysis are evaluated in terms of the extent to which the resulting trajectories cover an adequate range of scenarios, while the results of the second analysis are evaluated in terms of a range of outcomes, including the extent to which the priors for $D_{I}$ ('Year 25 depletion prior'), $K$ (the two carrying capacity priors), and $N_{c u r}$ are updated simply by projecting the model forward and rejecting parameter combinations that are not consistent with the assumed population dynamics.

\section{Population dynamics}

The model of the population dynamics is a deterministic Schaefer production model, i.e.:

$$
N_{t+1}=N_{t}+r N_{t}\left(1-N_{t} / \mathrm{K}\right)-\phi E_{t} N_{t} ; \quad N_{0}=K
$$

where $N_{t}$ is the population size at the start of year $\mathrm{t}, r$ is the intrinsic rate of growth (set to 0.05 , equivalent to an MSYR of $0.025^{7}$ ), $K$ is carrying capacity (set to the current abundance, $N_{75}=1,000$, divided by current depletion $\mathrm{D}_{75}$, i.e. $\left.K=1,000 / D_{75}\right), E_{t}$ is the relative effort in year $t$ (Fig. 1, column 1), and $\phi$ is a catchability parameter, selected so that the pre-specified depletion $D_{75}$ is achieved. The value of $\phi$ is set separately for each of the five effort scenarios. The term $\phi E_{t} N_{t}$ is replaced by the catch for year $t$ from the operating model when applying the Bayesian estimation methods.

\section{RESULTS AND DISCUSSION}

\section{Results of 'hitting' analyses}

Fig. 2 shows the time-trajectories of true population size (thick red line) for each of the five effort scenarios (rows) in

\footnotetext{
${ }^{5} 0.3$ is within the range of the CVs of the estimates of abundance used when conditioning operating models, but the results would be insensitive to this value.

${ }^{6}$ The post-model-pre-data distribution for a parameter is the distribution for that parameter that arises once parameter combinations that are inconsistent with the population dynamics model are excluded; post-model-pre-data distributions can be computed for model outputs (derived variables) as well as parameters.

${ }^{7}$ Results (not shown here) confirm that the qualitative results are insensitive to decreasing (to 0.02 ) or increasing (to 0.1 ) the value assumed for $r$.
} 
$(1,1)$

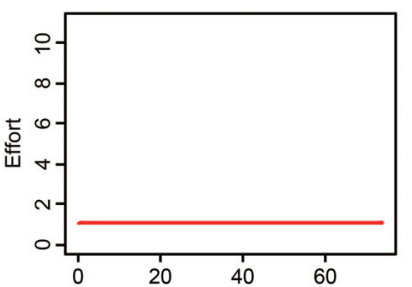

$(10,1)$

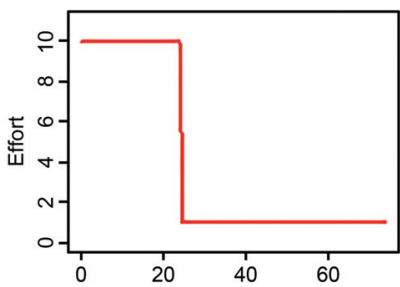

$(0.1,1)$
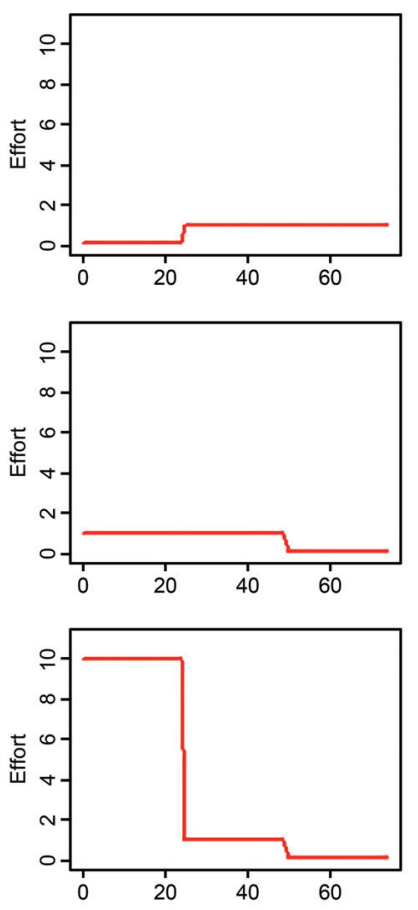

Depletion $=0.25$
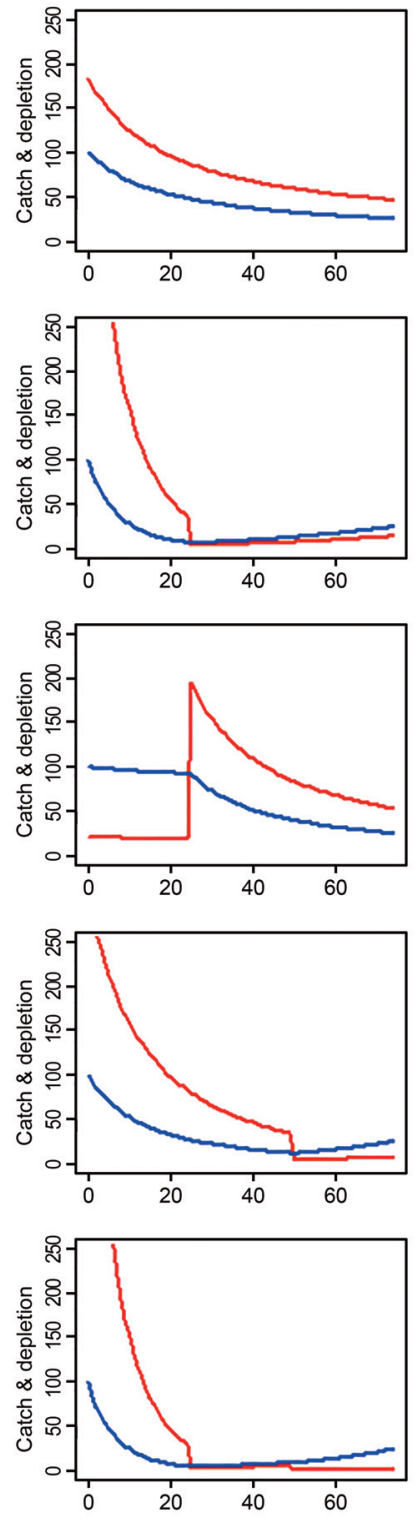

Depletion $=0.5$
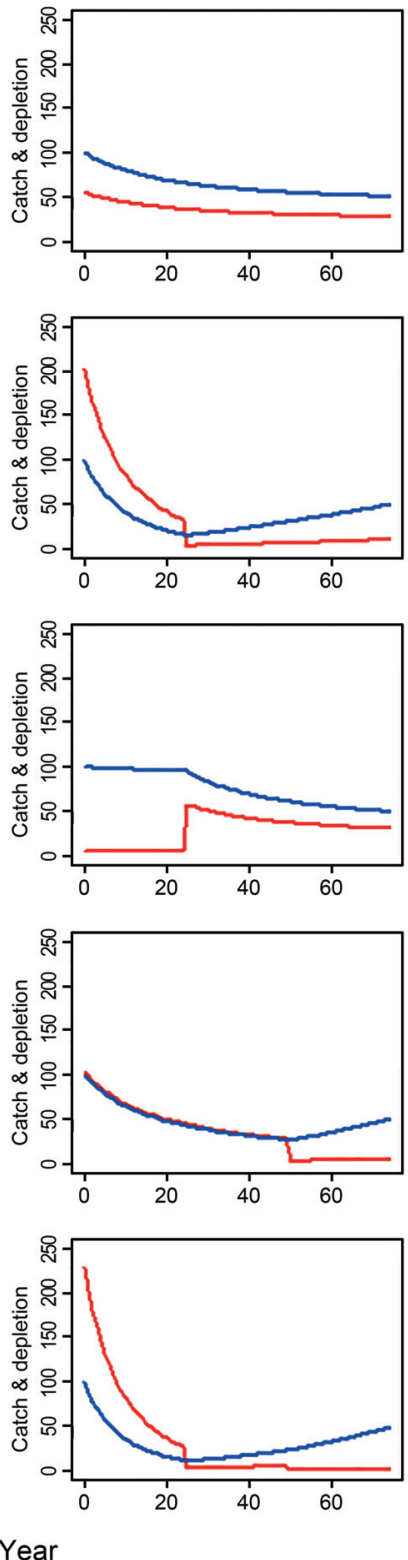
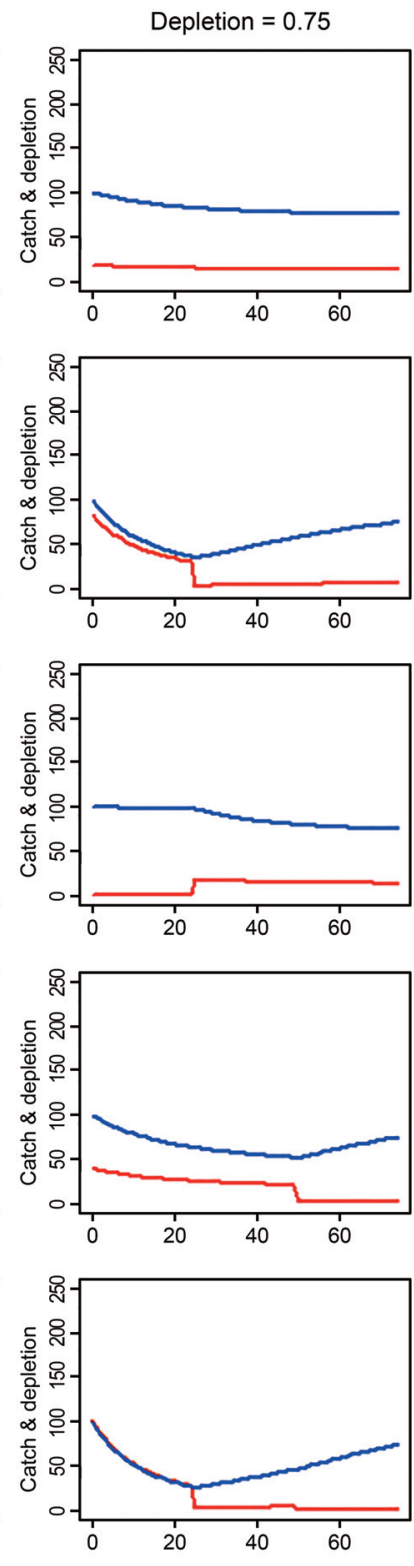

Fig. 1. Time-trajectories of effort (left column), and of catch [red lines] and depletion [blue lines] (columns 2-4). Results are shown for five scenarios regarding the time-trajectories of relative effort (rows).

absolute terms and relative to carrying capacity, along with the range of time-trajectories of inferred population size in absolute and relative terms for each of the three priors (columns). The results in Fig. 2 pertain to $N / K=0.5$ in year 75 (results for $N / K=0.25$ and $N / K=0.75$ are shown in supplementary Figs S.1 and S.2). As expected, all of the time-trajectories of inferred population size (the blue lines) pass through the 1,000 in year 75. The distributions of timetrajectories of inferred population sizes, in absolute terms, are quite tight irrespective of the method used to account for uncertainty. However, there is less variability in the timetrajectories of population size when the priors on carrying capacity are used, which is most noticeable for relative population size.

\section{Post-model-pre-data distribution}

Allowing for uncertainty in current abundance (log-normal with a log-scale standard deviation of $0.3 ; N / K$ in year 75 of
0.5 in Fig. 3 and of 0.25 and 0.75 in Supplementary Figs S.3 and S.4) led to results that are generally qualitatively similar to those when current abundance is known. However, carrying capacity prior (B) leads to samples from the prior that do not include the true trajectory for the 1-1 effort scenario and particularly the $0.1-1$ effort scenario for much of the time-series (first and third rows in Fig. 3). This discrepancy is related to the value of $N / K$ in year 75 , with the effect being smaller for $D_{75}=0.25$ and larger for $D_{75}=0.75$ (Supplementary Figs S.3 and S.4). None of the three methods do well at including the true trajectory in the sampled trajectories for the $0.1-1$ effort scenario, particularly for $D_{75}=0.75$ (Supplementary Fig. S.4).

The number of draws from the priors that are not consistent with the model (and are rejected in the process of constructing the post-model-pre-data distribution) differs among the various prior assumptions (Table 1). It is possible to find acceptable values for carrying capacity for almost all 

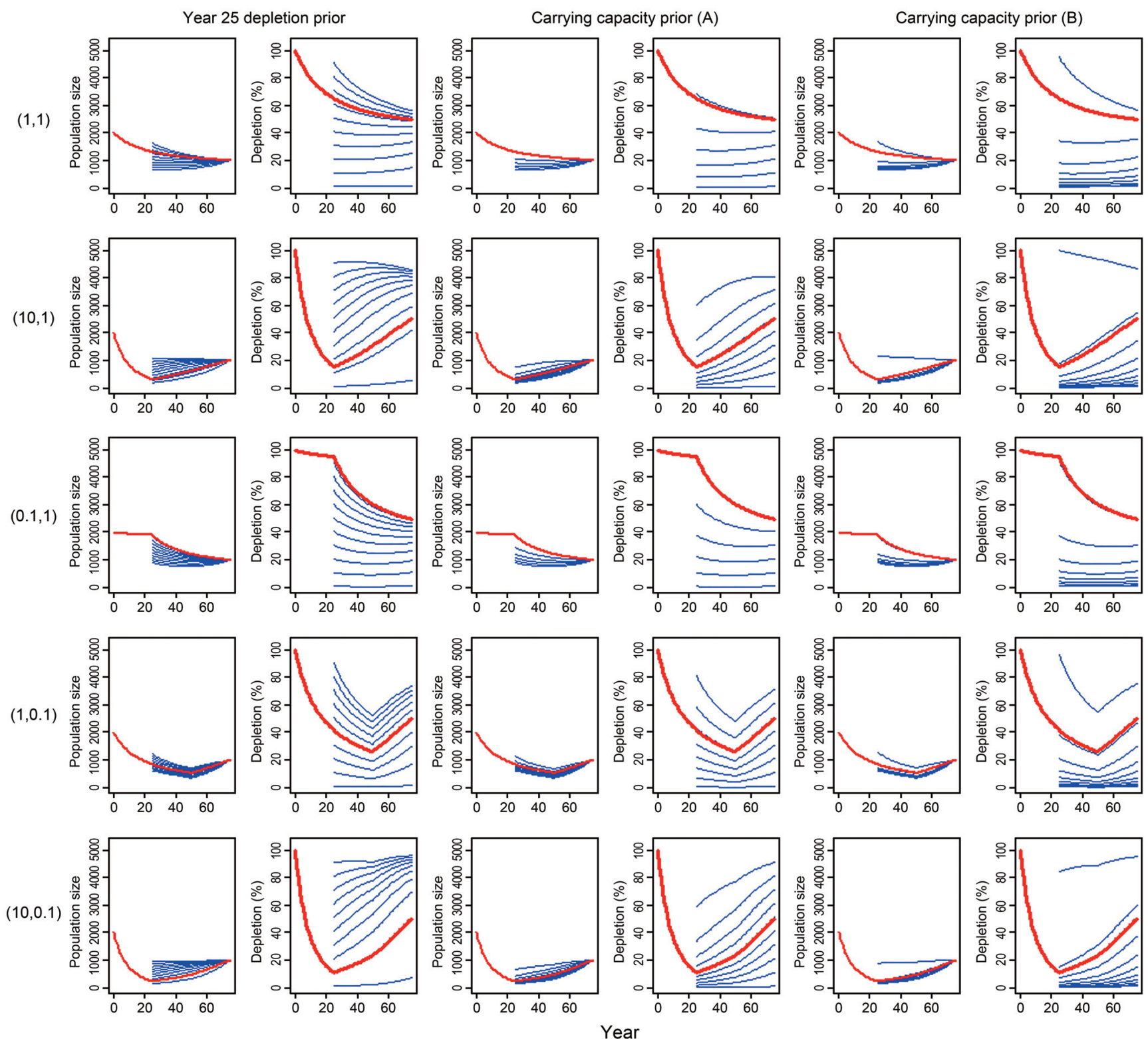

Fig. 2. Time-trajectories of true population size (thick red line) for each of the five effort scenarios (rows) in absolute terms and relative to carrying capacity (depletion), along with the range of time-trajectories of inferred population size (thin blue lines) in absolute and relative terms when sampling from each of the three priors (columns). The results in this figure pertain to $N / K=0.5$ in year 75 .

Table 1

Proportion of 100,000 draws from the prior that were not rejected as infeasible as a function of the relative population size (depletion) in year 75 and the effort scenario.

\begin{tabular}{cccc}
\hline $\begin{array}{c}\text { Relative population/ } \\
\text { effort scenarios }\end{array}$ & $\begin{array}{c}\text { Year 25 depletion } \\
\text { prior }\end{array}$ & $\begin{array}{c}\text { Carrying Capacity } \\
\text { prior (A) }\end{array}$ & $\begin{array}{c}\text { Carrying Capacity } \\
\text { prior (B) }\end{array}$ \\
\hline$N_{75} / K=0.25$ & & & 0.788 \\
1,1 & 0.996 & 0.375 & 0.961 \\
10,1 & 1.000 & 0.848 & 0.710 \\
$0.1,1$ & 0.986 & 0.257 & 0.902 \\
$1,0.1$ & 1.000 & 0.644 & 0.976 \\
$10,0.1$ & 1.000 & 0.908 & \\
$N_{75} / K=0.5$ & & & 0.883 \\
1,1 & 1.000 & 0.589 & 0.965 \\
10,1 & 1.000 & 0.865 & 0.856 \\
$0.1,1$ & 1.000 & 0.513 & 0.943 \\
$1,0.1$ & 1.000 & 0.784 & 0.976 \\
$10,0.1$ & 1.000 & 0.910 & \\
$N_{75} / K=0.75$ & & & 0.948 \\
1,1 & 1.000 & 0.803 & 0.970 \\
10,1 & 1.000 & 0.885 & 0.942 \\
$0.1,1$ & 1.000 & 0.782 & 0.963 \\
$1,0.1$ & 1.000 & 0.857 & 0.977 \\
$10,0.1$ & 1.000 & 0.911 & \\
\hline
\end{tabular}


values for year 25 depletion (the few exceptions being somewhat surprisingly - the constant effort scenario). In contrast, up to $29 \%$ of the 'Carrying capacity prior (B)' draws can be rejected, but it is generally possible to find values for initial population size to match the generated current abundance and carrying capacity values. The poorest performance in terms of rejecting draws during the postmodel-pre-data stage is 'Carrying capacity prior (A)' for which it is seldom possible to find an initial depletion that matches the generated current abundance and carrying capacity values, and the proportion of rejected draws can be as high as $74 \%$, with most of the rejections occurring for higher levels for current depletion (i.e. population sizes close to carrying capacity). Although a large proportion of draws may be rejected, this does not (visually at least) impact the post-model-pre-data distribution for current abundance (Fig. 4).
Fig. 5 shows the post-model-pre-data distribution for initial (year 25) and current (year 75) depletion. The distributions (except for initial depletion for the 'Year 25 depletion prior') are far from uniform, suggesting that any of the choices of prior lead to a priori implications regarding stock status at various points in time. The sensitivity of the post-model-pre-data distributions for current depletion to the effort scenario is greatest for the 'Year 25 depletion prior, with three of the distributions centered somewhere close to the true depletion and two centered on much more optimistic values (Fig. 5, column 2). In contrast, 'Carrying capacity prior (A)' implies that the stock was fairly depleted in year 25 irrespective of the effort scenario. The distribution for current depletion is shifted towards lower values for this prior, even though this method was developed based on a uniform distribution for current depletion. 'Carrying capacity prior (B)' leads to the most
Year 25 depletion prior

$(1,1)$
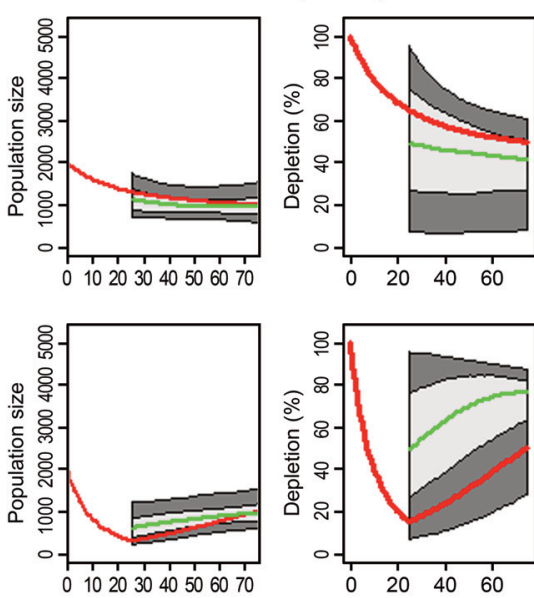

$(0.1,1)$
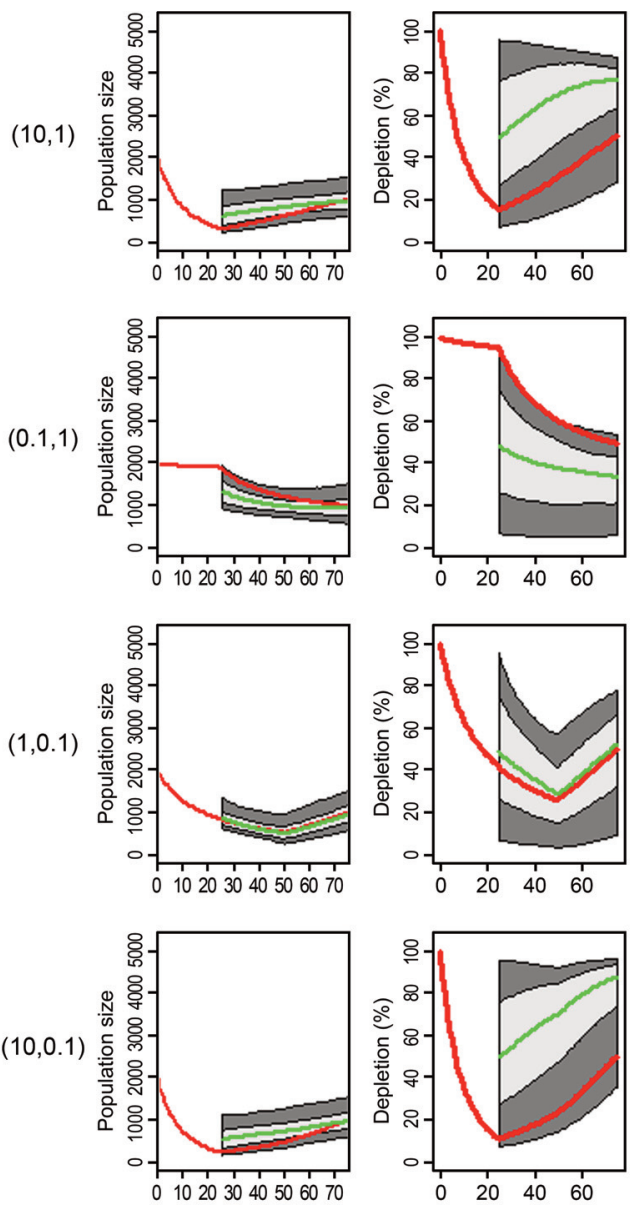
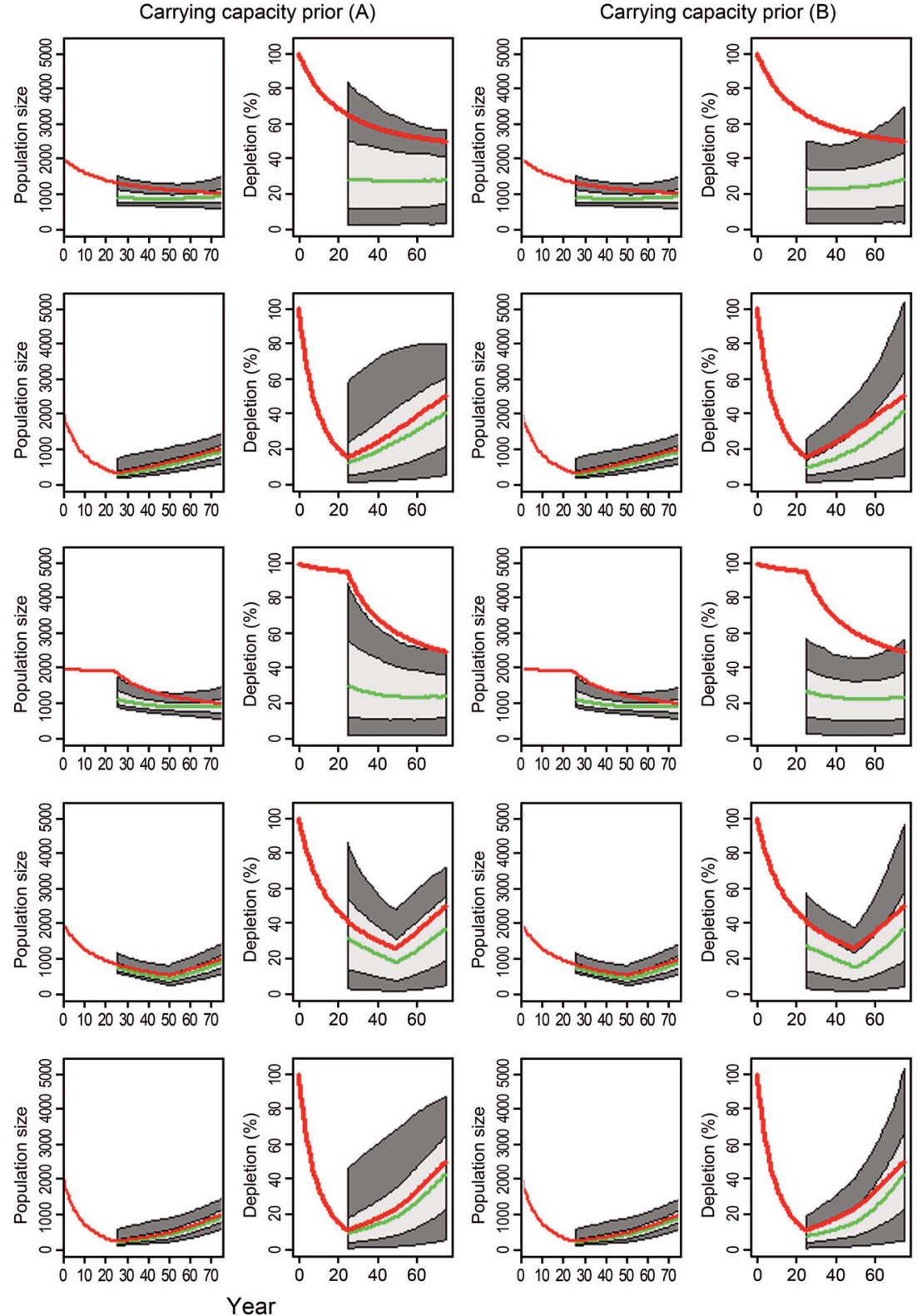

Fig. 3. Time-trajectories of true population size (thick red line) for each of the five effort scenarios (rows) in absolute terms and relative to carrying capacity (depletion), along with the range of time-trajectories of inferred population size in absolute and relative terms for each of the three priors when account is taken of uncertainty in current population size (columns). The green lines denote the distribution medians, the light gray shading the $50 \%$ iles of the distributions and the dark gray shading the $90 \%$ iles of the distributions. The results in this figure pertain to $N / K=0.5$ in year 75 . 
$(1,1)$
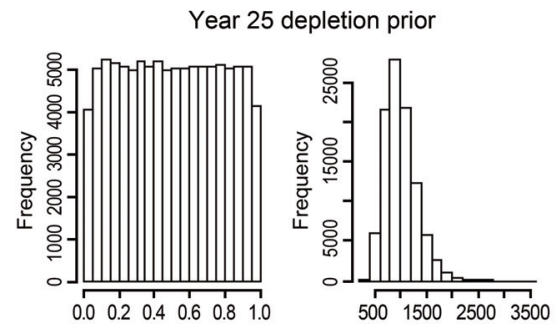

$(10,1)$
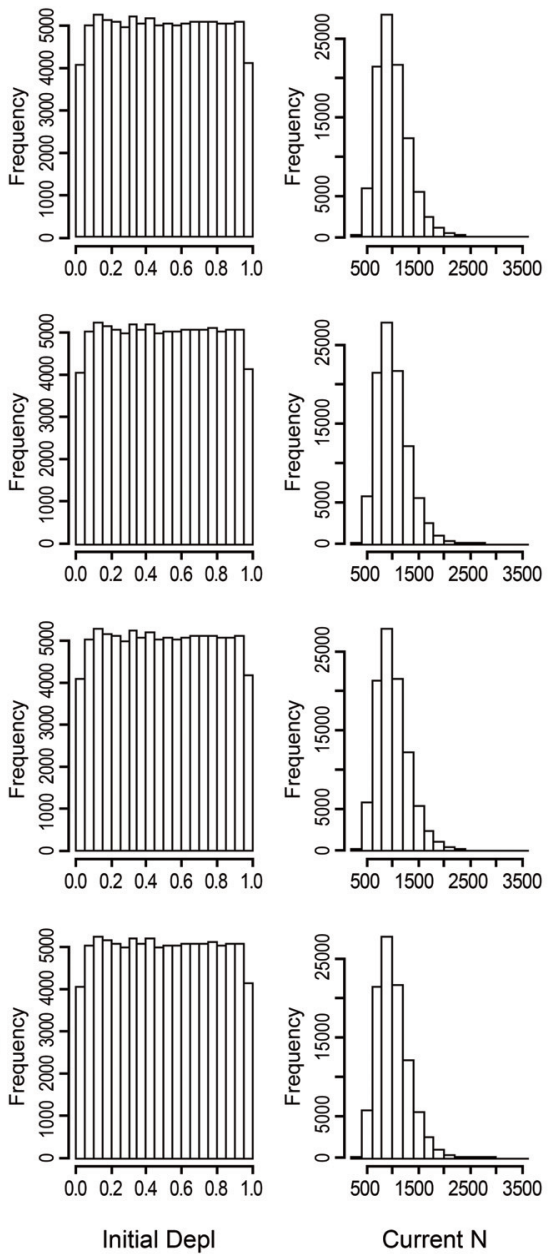
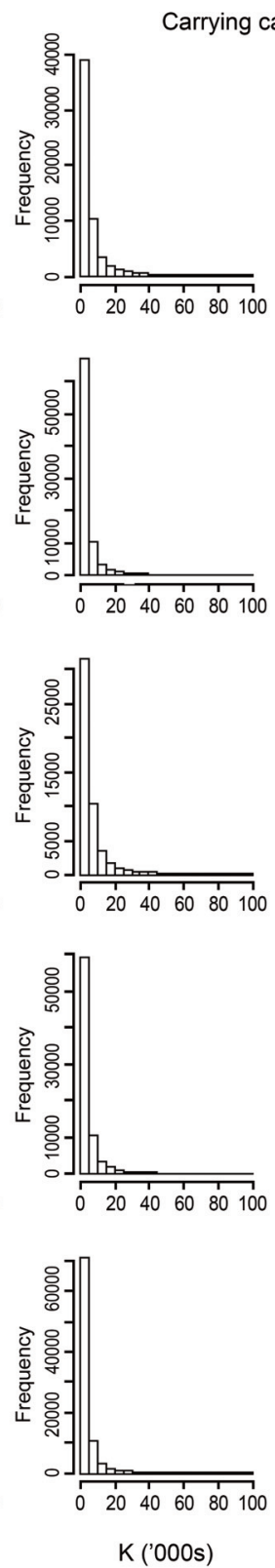
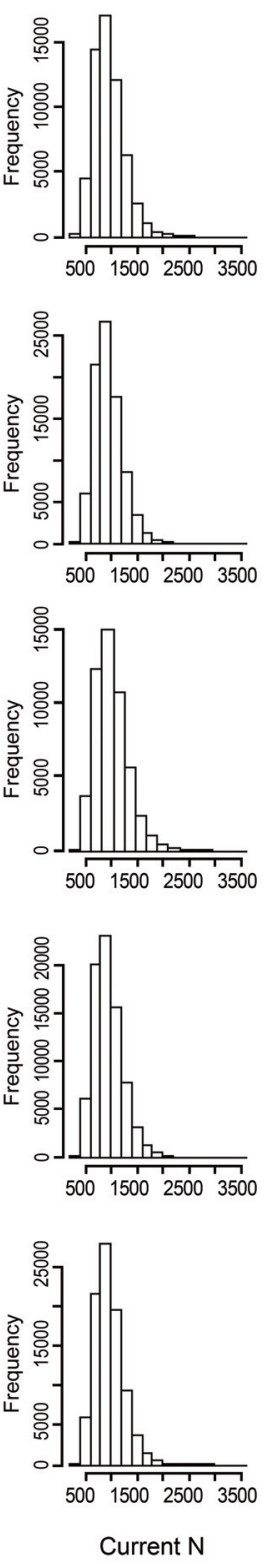

Carrying capacity prior (B)
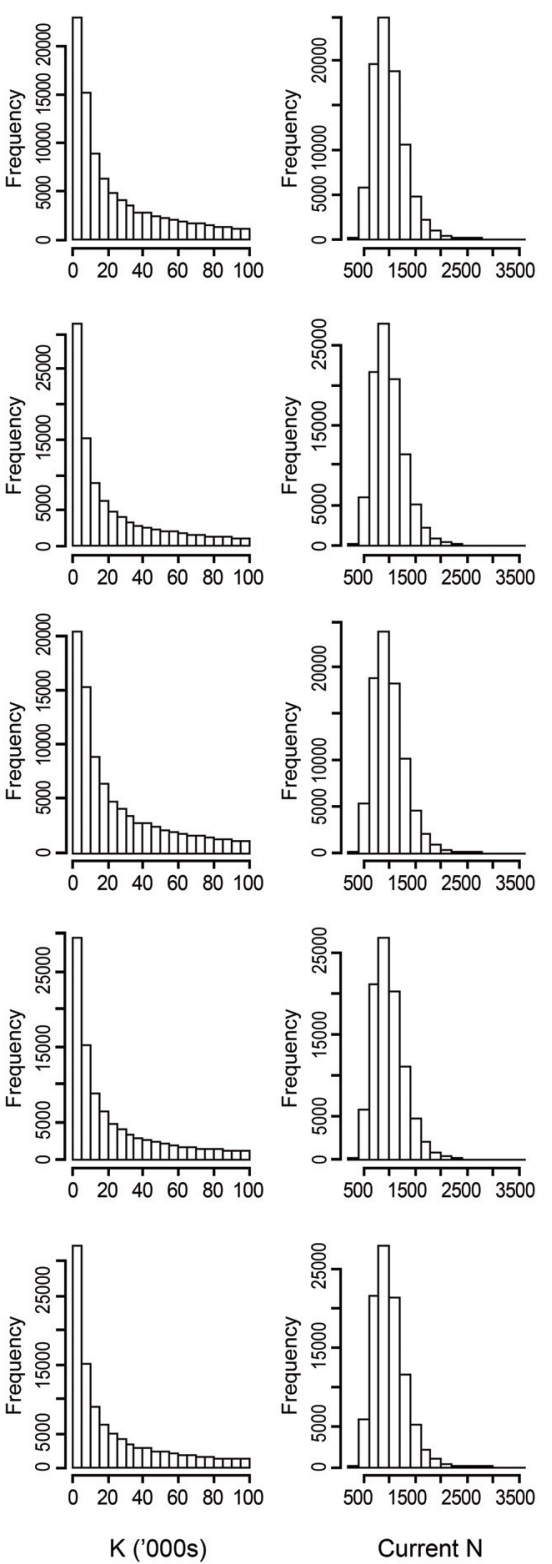

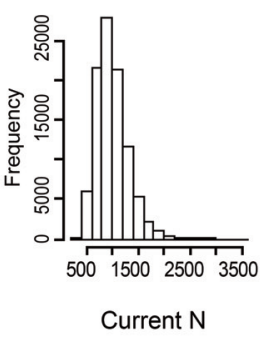

Fig. 4. Histograms of the post-model-pre-data distribution for the variable that is generated to create the scaling parameter (first column for each prior) and the post-model-pre-data distribution for population size in year 75 . The results in this figure pertain to $N / K=0.5$ in year 75 . The results for other values of $N / K$ in year 75 are very similar and are not shown.

extreme depletion distributions, with the implication a priori that the stock was highly depleted and still is, irrespective of how effort (and hence fishing mortality) has changed over time.

\section{CONCLUSIONS, CAVEATS AND FINAL REMARKS}

The process of constructing the post-model-pre-data distribution has long been known to lead to rejected parameter combinations (e.g. Brandon et al., 2007). However, those analyses tended to focus on biological parameters such as survival and the age-at-maturity. The analysis of this paper suggests that the choice of the prior for the 'scaling' parameter (carrying capacity or initial depletion) has a large impact on the proportion of rejected draws and that the resulting post-model-pre-data distributions are sensitive to the choice of the scaling parameter and the prior assigned to it (and for 'year 25 depletion prior' to the series of historical catches). In the case of 'Carrying capacity prior (A)' the post-model-predata distribution for the parameter on which the prior is based is updated, sometimes quite substantially, which is undesirable because priors should be only be updated due to data.

The effects found here will, of course, be 'mitigated' by the availability of data, as the data will update the priors, and the 'scaling' parameter should be updated substantially if there is a time series of estimates of abundance. Nevertheless, some IWC MSEs (e.g. for West Greenland bowhead whales) are based on limited data, and the extent of updating may be low. This can have an effect on the final outcomes. For example, 'carrying capacity prior (B)' implies higher levels of carrying capacity than the other two priors. As MSY is the product of $r$ and $K$, this implies higher MSY for this choice of prior (which is close to the default for recent IWC MSEs for aboriginal subsistence whaling operations) and hence that more 'aggressive' Strike Limit 
Year 25 depletion prior

$(1,1)$
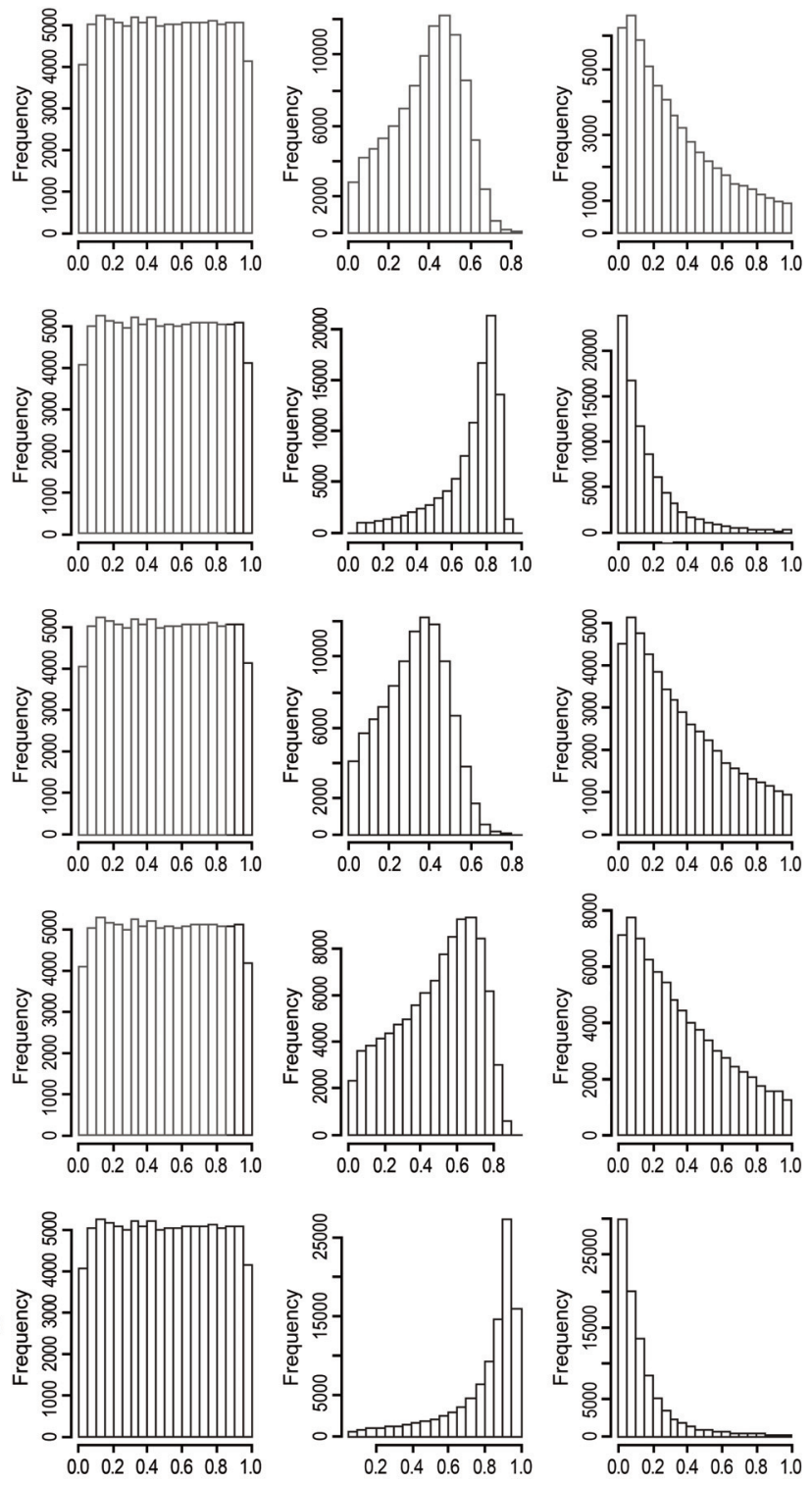

Current Dep
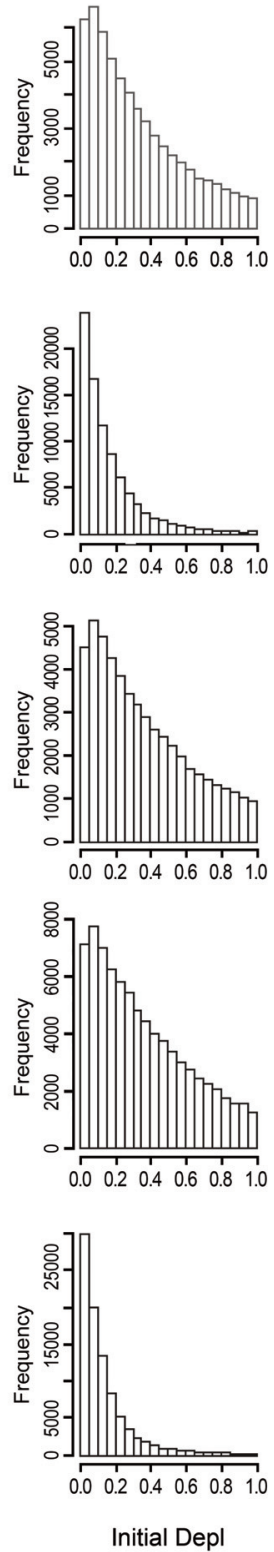
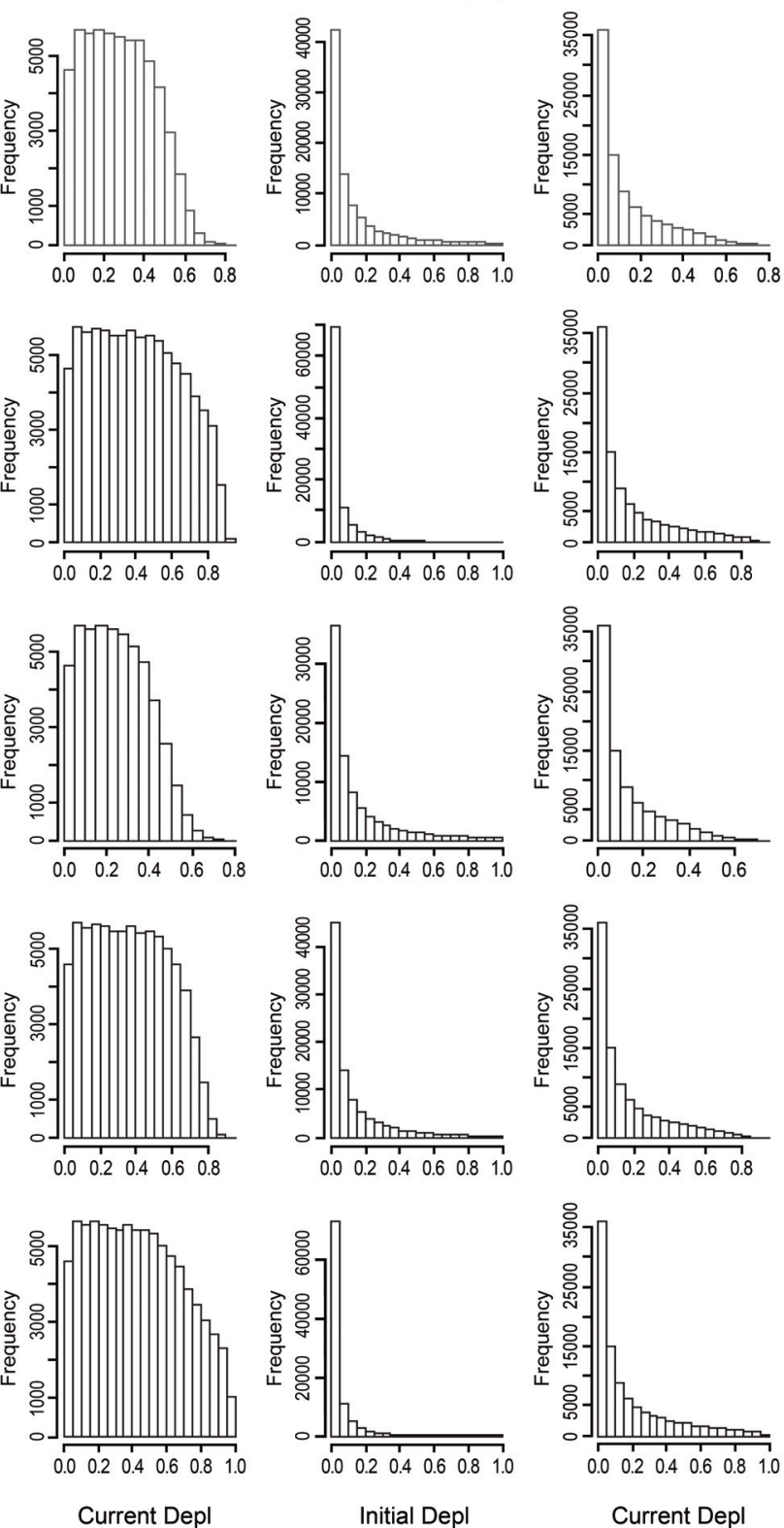

Fig. 5. Histograms of the post-model-pre-data distribution for initial (year 25) and current (year 75) relative population size (depletion). The results in this figure pertain to $N / K=0.5$ in year 75 . The results for other values of $N / K$ in year 75 are very similar and are not shown.

Algorithms could be concluded to be 'safe' from a conservation viewpoint.

The analyses of this paper are based on a simple population dynamics model and actual simulation trials are usually based on more complex (e.g. sex- and age-structured, and often spatial) models. These models contain additional reasons for rejecting draws from prior distributions (e.g. parameter combinations that lead to adult survival being lower than that for juveniles). However, the general concerns raised here would be robust to more complex model structures because the fundamental population dynamic process that leads to the results (the $r-K$ trade-off) is a basic property of all population models.

Overall, the results of this paper lead to some recommendations regarding future best practices for Bayesian MSEs (particularly those for which data are sparse and/or uninformative) as well as Bayesian and Bayesian-like data-poor methods for assessment stocks (e.g. Martell and Froese, 2013).
(1) The issue of the appropriate distribution for the 'scaling' parameter should be an explicit component of the IWC Implementation and Implementation Review process, and in general in best practice guidelines for MSE (e.g. Punt et al., 2016).

(2) The post-model-pre-data distribution (and posterior) for key parameters (such as initial and final depletion and MSY) should be reported and evaluated, particularly if the prior distribution for the scaling parameter is not updated substantially. It is necessary to assess the extent to which the posterior for key model outputs reflects the data or the prior/the post-model-pre-data distribution.

(3) There may be value in 'redrawing' parameters when the implied post-model-pre-data distribution does not match that intended (c.f. Brandon et al., 2007).

(4) There would be value in considering more than one assumption (e.g. the three approaches considered in this 
paper) regarding the 'scaling' parameter in MSEs if the post-model-pre-data distributions (and posteriors) are sensitive to the choice of 'scaling parameter'.

\section{ACKNOWLEDGEMENTS}

Gisli Vikingsson (Marine and Freshwater Research Institute, Iceland) is acknowledged for noting during an AWMP meeting in Copenhagen surprise that all simulation trials led to very low initial depletions when using a 'Carrying capacity prior (B)'-like prior. I apologise for taking so long before being to address the issue. Natalie Dowling (CSIRO), Malcolm Haddon (CSIRO, Retired), and two anonymous reviewers are thanked for their comments on an earlier version of this paper.

\section{REFERENCES}

Brandon, J.R., Breiwick, J.M., Punt. A.E. and Wade, P.R. 2007. Constructing a coherent joint prior while respecting biological bounds: application to marine mammal stock assessments. ICES J. Mar. Sci. 64: 1085-1100.

Bunnefeld, N., Hoshino, E. and Milner-Gulland, E. J. 2011. Management strategy evaluation: a powerful tool for conservation? Trends in Ecol. Evol. 26: 441-7.

Dichmont, C.M., Ellis, N., Bustamante, R.H., Deng, R., Tickell, S., Pascual, R., Lozano-Montes, H. and Griffiths, S. 2013. Evaluating marine spatial closures with conflicting fisheries and conservation objectives. J. Appl. Ecol. 50: 1060-70

Fulton, E.A., Punt, A.E., Dichmont, C.M., Harvey, C.J. and Gorton, R. 2019. Ecosystems say good management pays off. Fish and Fish. 20: 66-96.

Hillary, R.M., Preece, A.L., Davies, C.R., Kurpta, H., Itoh, T., Parma, A.M., Butterworth, D.S., Ianelli, J. and Branch, T.A. 2016. A scientific alternative to moratoria for rebuilding depleted international tuna stocks. Fish and Fish 17: 469-2.
International Whaling Commission. 2003. Report of Standing Working Group on the Aboriginal Subsistence Whaling Management Procedure. J. Cetacean Res. Manage. (Suppl.) 5: 154-225.

International Whaling Commission. 2012. The Revised Management Procedure (RMP) for Baleen Whales. J. Cetacean Res. Manage. (Suppl.) 13: 485-94

International Whaling Commission. 2014a. Report of Sub-Committee on the Revised Management Procedure. J. Cetacean Res. Manage. (Suppl.) 15: $87-188$.

International Whaling Commission. 2014b. Report of the Standing Working Group on the Aboriginal Subsistence Whaling Management Procedure. J. Cetacean Res. Manage. (Suppl.) 15: 189-213.

International Whaling Commission. 2014c. Report of the Scientific Committee. J. Cetacean Res. Manage. (Suppl.) 15: 1-75

International Whaling Commission. 2017. Report of Sub-Committee on the Revised Management Procedure. J. Cetacean Res. Manage. (Suppl.) 18: 123-73.

International Whaling Commission. 2017a. Report of Sub-Committee on the Revised Management Procedure. J. Cetacean Res. Manage. (Suppl.) 20: 93-119.

International Whaling Commission. 2019b. Report of the Standing Working Group on the Aboriginal Subsistence Whaling Management Procedure. J. Cetacean Res. Manage. (Suppl.) 20: 120-82.

International Whaling Commission. 2019c. Report of the Fifth Rangewide Workshop on the Status of North Pacific Gray Whales, 28-31 March 2018, SWFSC, Big Sur, California, USA. J. Cetacean Res. Manage. (Suppl.) 20: 569-99.

Martell, S. and Froese, R. 2013. A simple method for estimating MSY from catch and resilience. Fish and Fisheries 14: 504-14.

Plagányi. É.E., Rademeyer, R.A., Butterworth, D.S., Cunningham, C.L. and Johnston, S.J. 2007. Making management procedures operational innovations implemented in South Africa. ICES J. Mar. Sci. 64: 626-32.

Punt, A.E. and Donovan, G. 2007. Developing management procedures that are robust to uncertainty: Lessons from the International Whaling Commission. ICES J. Mar. Sci. 64: 603-12.

Punt, A.E., Butterworth, D.S., de Moor, C.L., De Oliveira, J.A.A. and Haddon, M. 2016. Management Strategy Evaluation: Best Practices. Fish and Fisheries 17: 303-34

[Supplementary Figures are on following pages] 

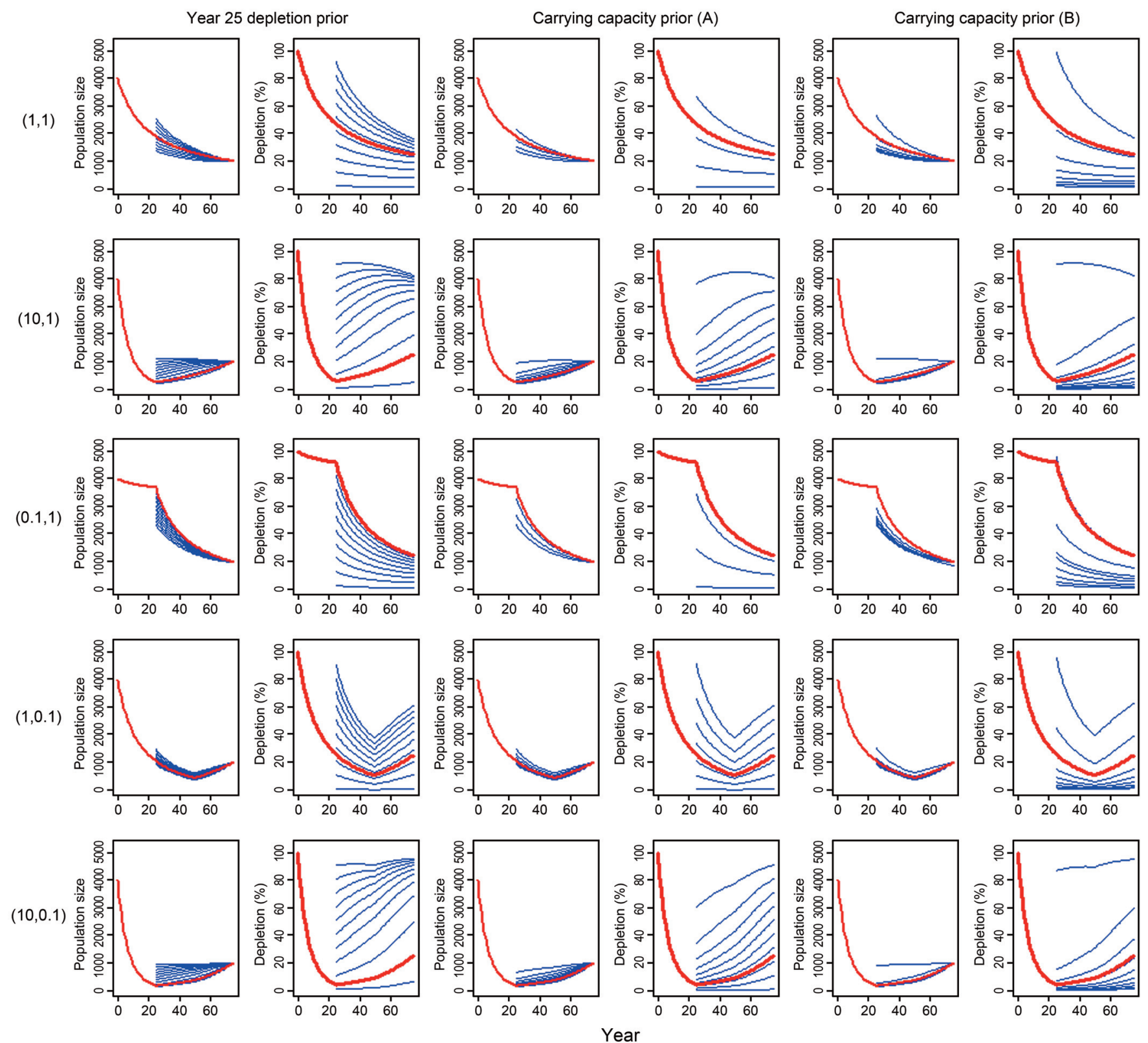

Supplementary Fig. S.1. Time-trajectories of true population size (thick red line) for each of the five effort scenarios (rows) in absolute terms and relative to carrying capacity, along with the range of time-trajectories of inferred population size in absolute and relative terms (thin blue lines) for each of the three priors (columns). The results in this figure pertain to $N / K=0.25$ in year 75 . 

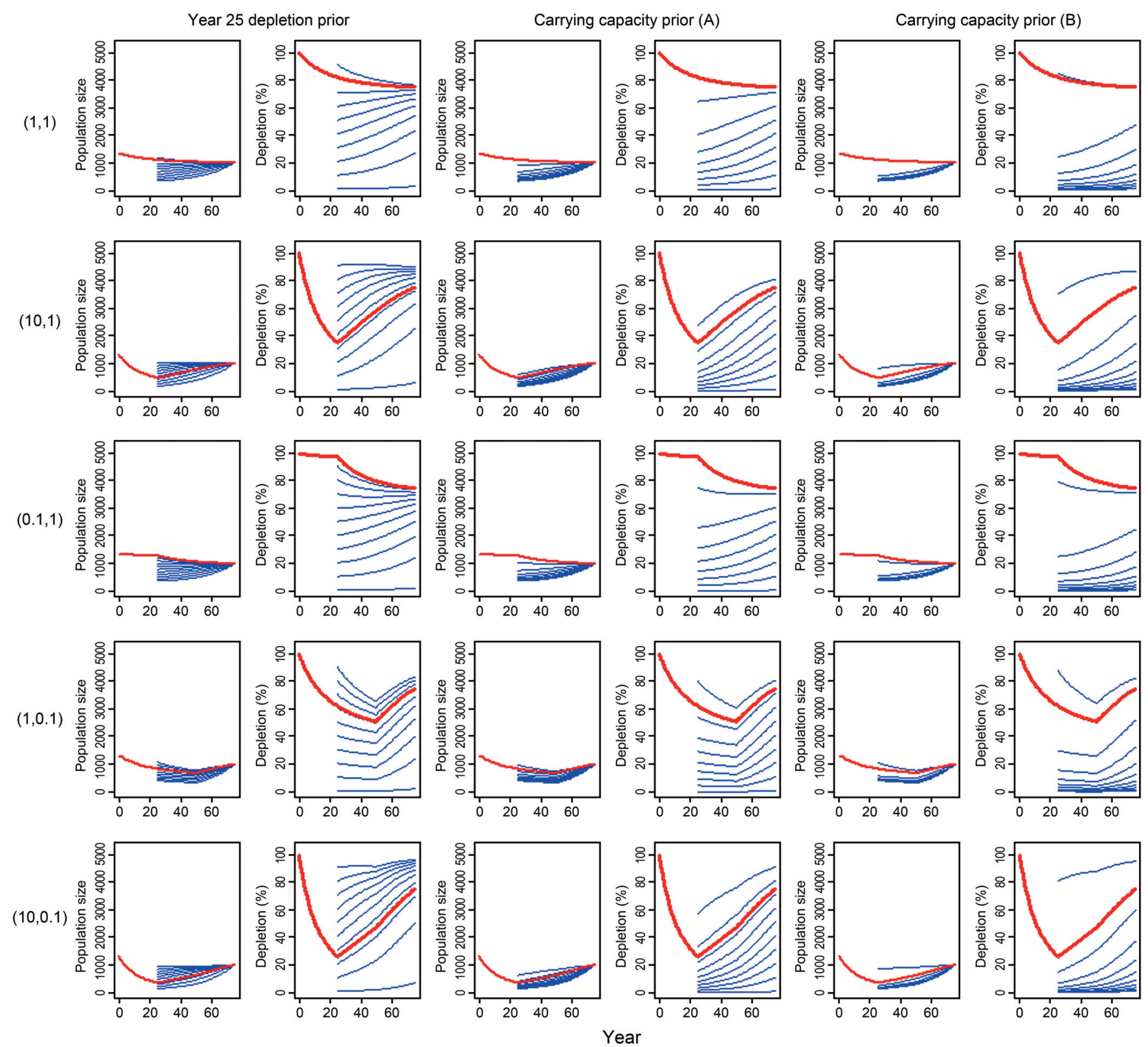

Supplementary Fig. S.2. Time-trajectories of true population size (thick red line) for each of the five effort scenarios (rows) in absolute terms and relative to carrying capacity, along with the range of time-trajectories of inferred population size in absolute and relative terms (thin blue lines) for each of the three priors (columns). The results in this figure pertain to $N / K=0.75$ in year 75 . 
$(1,1)$
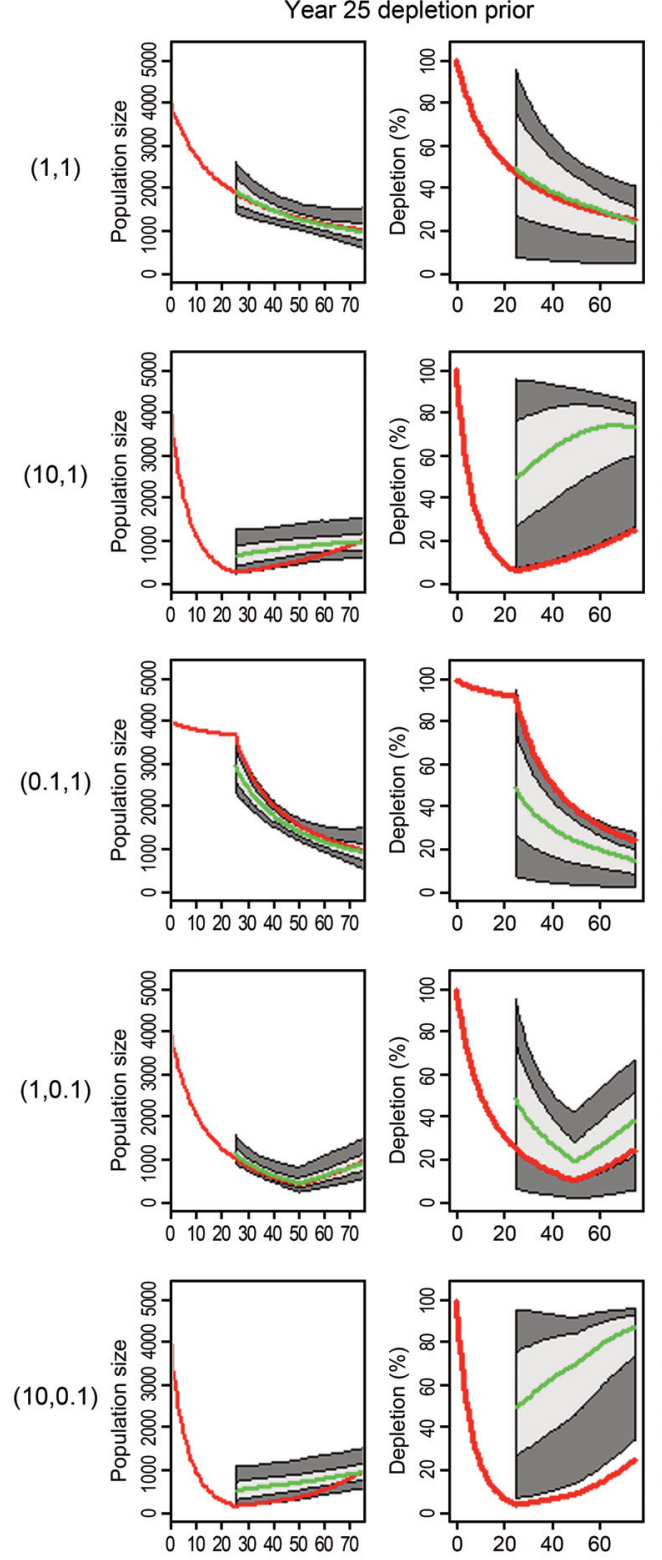
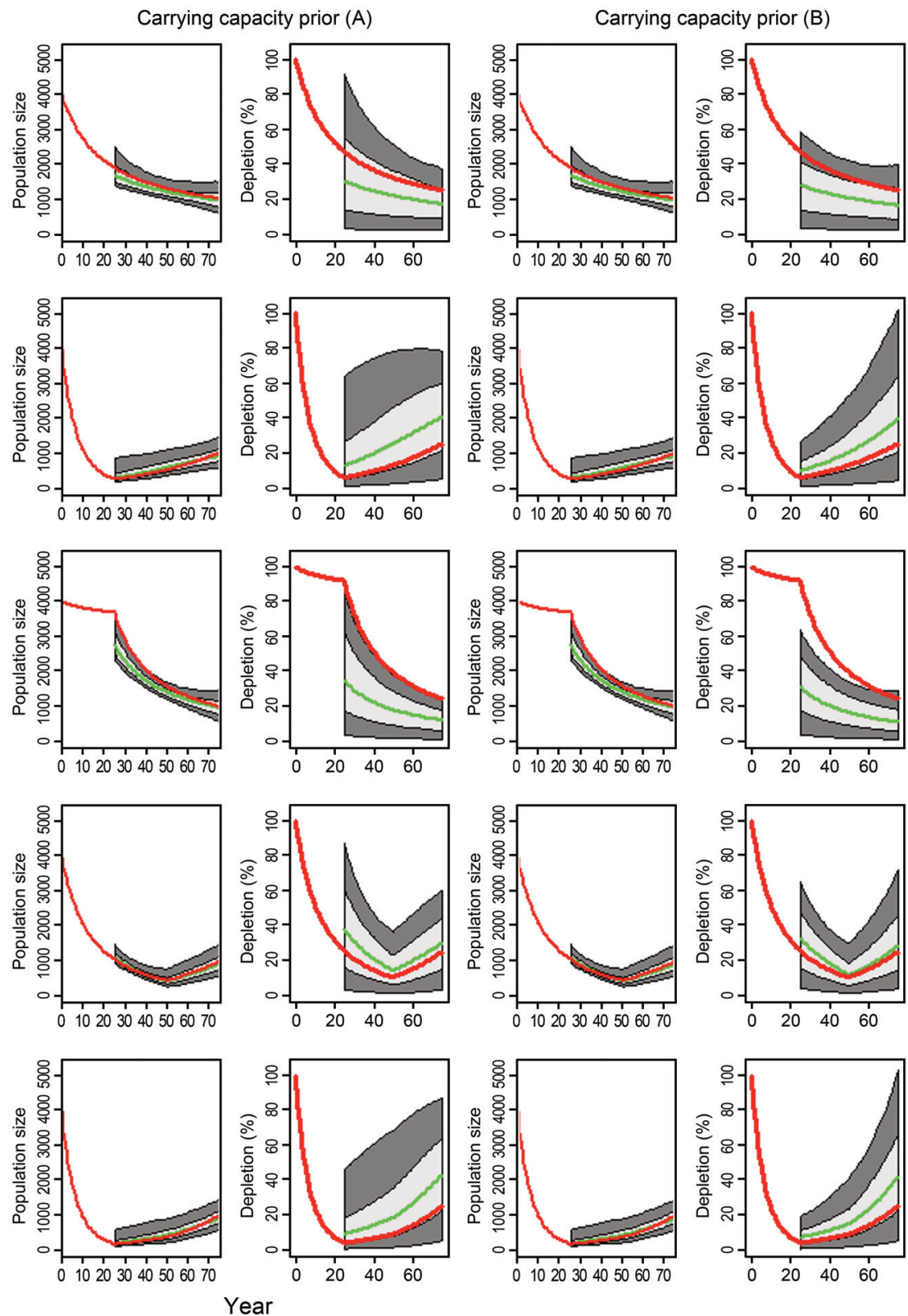

Supplementary Fig. S.3. Time-trajectories of true population size (thick red line) for each of the five effort scenarios (rows) in absolute terms and relative to carrying capacity, along with the range of time-trajectories of inferred population size in absolute and relative terms for each of the three priors when account is taken of uncertainty in current population size (columns). The green lines denote the distribution medians, the light grey shading the $50 \%$ iles of the distributions and the dark grey shading the $90 \%$ iles of the distributions. The results in this figure pertain to $\mathrm{N} / \mathrm{K}=0.25$ in year 75 . 

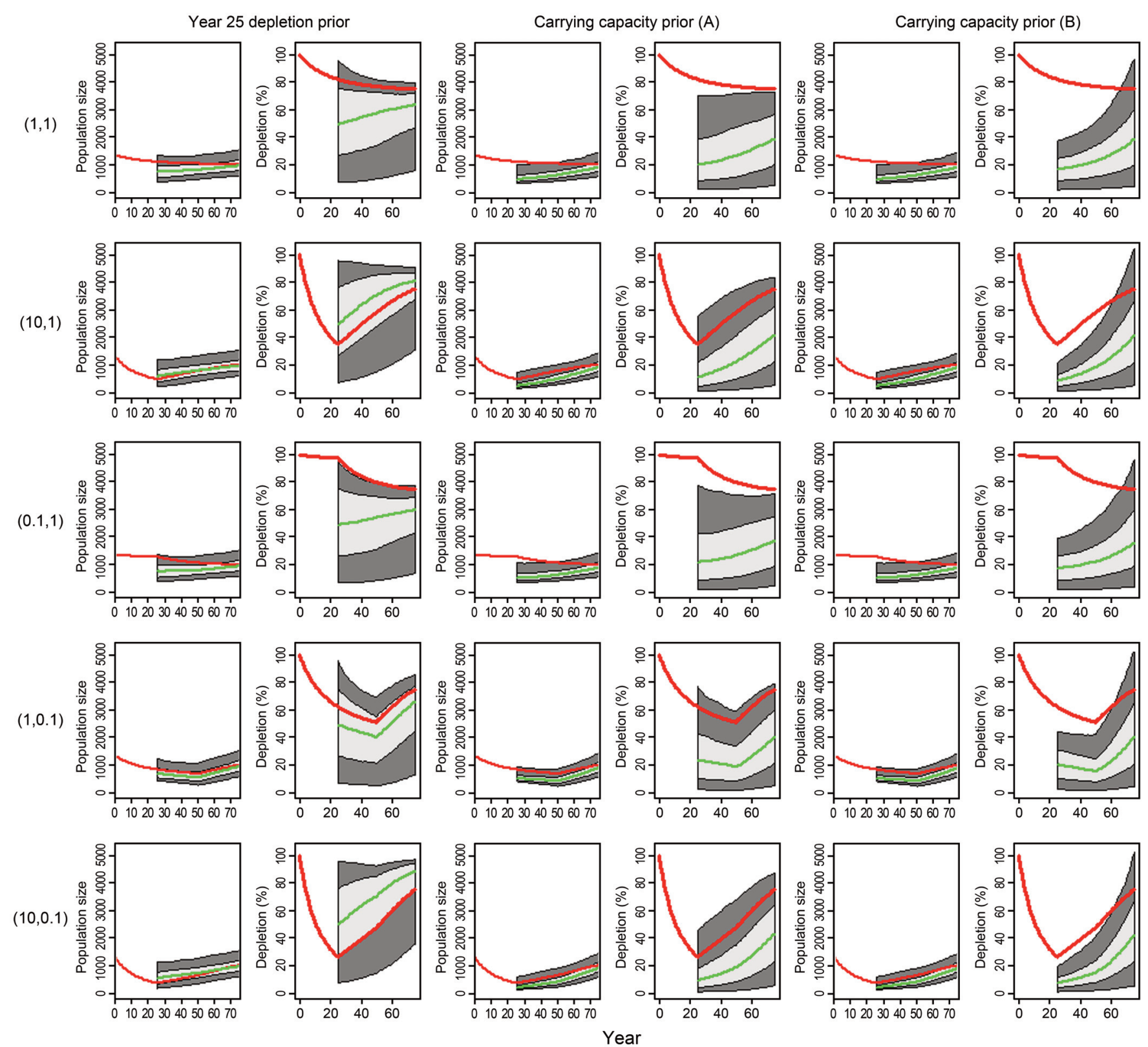

Supplementary Fig. S.4. Time-trajectories of true population size (thick red line) for each of the five effort scenarios (rows) in absolute terms and relative to carrying capacity, along with the range of time-trajectories of inferred population size in absolute and relative terms for each of the three priors when account is taken of uncertainty in current population size (columns). The green lines denote the distribution medians, the light grey shading the $50 \%$ iles of the distributions and the dark grey shading the $90 \%$ iles of the distributions. The results in this figure pertain to $\mathrm{N} / \mathrm{K}=0.75$ in year 75 . 